\title{
IMPLICATION AS AN ALTERNATIVE TO SET-INCLUSION \\ AS THE SEMANTIC PRIMITIVE
}

Arnold Lewis Glass

Stanford University

The componential analysis of language as an approach to semantics has a long, rich history spanning several disciplines, including philosophy, linguistics, and psychology. The basic idea is that a word can be broken down into a set of primitive properties (or ideas, features, markers, etc.) that can then be said to constitute its meaning. For example, man may be intuitively analyzed as the set of properties (male, human); and perhaps the property "human" may be further analyzed as (intelligent, mammal), etc. These properties (as they will henceforth be called) are ultimately represented as atomic, unitary, and independent particles. They may be thought of as a collection of uniquely colored marbles, where any assortment is possible.

The assumption of independent properties is common to a wide variety of substantially different theories which have appeared in different disciplines. Katz and Fodor (1963) use markers and distinguishers to disambiguate the meaning of a word in the context of a particular sentence. Schaffer and Wallace (1970) and Meyer (1970) have proposed setinclusion models to explain the correlation between the time it takes to verify whether a word is a member of a certain semantic category and the "semantic distance" between that word and the category. For example, it takes longer for a subject to judge "true" in response to the statement "a canary is an organism" than it takes to verify "a canary is a bird". The logical decision in these set-inclusion models rests on the presence of certain properties in the intersection of the sets of properties defining words. The union of the sets is searched for a property in the intersection. Hence, speed of verification is correlated with the amount of overlap between sets. As a final example of componential approaches, programs that attempt to simulate human language comprehension (e.g. . Shank, 1972; Winograd, 1972) utilize a priori defined primitives or properties.

The Limitations of a Componential Analysis

While componential analysis has been widely applied, it has limitations. If the question is asked, "Is an elm a tree?", the answer is sought in a componential model by searching a set labelled elm for a property labelled "tree", or equivalently, following an arrow from a node labelled elm to a property labelled "tree". This is certainly logically sufficient. But from a developmental point of view, how did these nodes get formed and their set-relations get specified in the first place? There are several possible answers to this question. 
One answer is that the components stand for independent perceptual features. In this version of the theory, a very small set of perceptual features characterize the set defining the concept "plant", a superset of it characterizes "tree", and a superset of "tree" characterizes "elm". Since a small set of perceptual features are contained in the set for "plant", a large set of objects will have all the perceptual features of "plant" . Some of the objects having all of the perceptual features of "plant" will have all of the perceptual features of "tree". Hence, it can be deduced from these definitions that the set of objects called plants includes the set of objects called trees; or, conversely, all trees are plants. Thus, logical implication is defined in terms of perceptual features. However, while the perceptual relationship that exists between trees and plants is certainly an instance of the application of logical implication, there are problems with making it the definition of logical implication, since there are other uses of logical implication where perceptual similarity does not appear relevant. It does not appear that a whale is classed with a dog (as a mammal) instead of a shark (as a fish) on the basis of perceptual similarity. Benches are classed with chairs on the basis of their function as something to sit on rather than with tables on the basis of perceptual similarity. And the statements "All men are mortal" and "p and $q$ implies $p^{\prime \prime}$ seem to have no interpretation in terms of perceptual similarity at all. For this reason, defining components solely in terms of perceptual features appears inadequate.

A second answer is to define a set of "primitive" components which express logical relationships and are somehow at a person's disposal due to innate mechanisms. Candidates for such primitives have run from "object" and "property" to "agent", "causality", and "implication". Postulating a primitive, of course, does not explain it, but only describes what the theory can't explain. Asserting the existence of a primitive is only an empirical claim to the extent which the relation of the primitive to the rest of the system is welldefined and it is seriously argued that the primitive represents something which underlies all human thought.

A third answer is to make the sets of properties more abstract so that they can represent any relationship which can be stated in natural language. In this case a system of rules must be stated which generate this representation and relate it back to sensory data which reference the external world. This is the approach that will be taken in this paper. No concepts like the ones mentioned above will be assumed to be innate. A formal representation on which logical decisions can be made will be defined; and it will briefly be shown how this representation can be generated by experience and related to natural language. From one point of view, it may be considered that this model is a refutation of componential models in the narrowest sense of the term, since the axioms used to generate the representation go beyond set-theoretic axioms. However, the model may also be seen as an extension 
of traditional componential work, taking up a question which past theorists weren't always concerned with: how the representation is generated.

Logical Implication Between Properties.

Almost any concept can be further discriminated into an arbitrary number of subconcepts. "Colored" can be discriminated into "red" and "green", "green" in turn may be discriminated into "kelly" and "forest". "Teeth" may be discriminated into "mechanical teeth" and "animal teeth", "animal teeth" may be discriminated into "shark teeth" and "human teeth", and "human teeth" may be discriminated into "molars" and "incisors". It does not seem likely that when a word is used, implicit in the underlying concept is all the subconcepts which can later be thought up. From this point of view, a concept is not ambiguous, but it is vague. Each subconcept is related to its superordinate by logical implication. Thus, "molar" implies "human teeth", "human teeth" implies "animal teeth", and "animal teeth" implies "teeth". A concept tree is shown in Figure 1. An entire tree will be referred to as a concept while each node on the tree will be referred to as a property. For

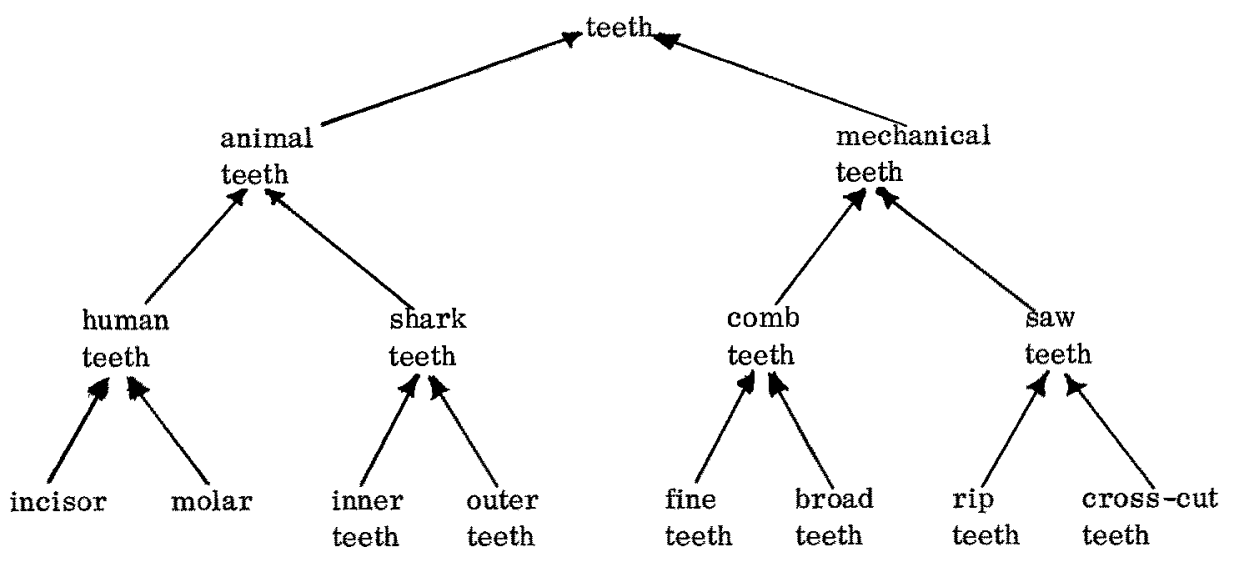

FIG. 1. The Representation of the Concept Tree for "Teeth".

example, in Figure 1, the concept "teeth" includes the entire tree. The concept "animal teeth" includes the properties "animal teeth", "shark teeth", "human teeth", "inner teeth", "outer teeth", "molars", and "incisors". The concept "human teeth" includes the properties "human teeth", "molars", and "incisors". The use of the term property here for a node on a tree is entirely consistent with the earlier use as the component of semantic analysis, since they will eventually be shown to be the same thing.

Two concept trees can intersect and have a property in common. The concept tree for "male" and the concept tree for "human" intersect at the property "man". Then, since 
both trees include the property, "man" implies both "male" and "human". Similarly, the property "animal teeth" implies both "animal" and "teeth". These examples are diagrammed in Figure 2.

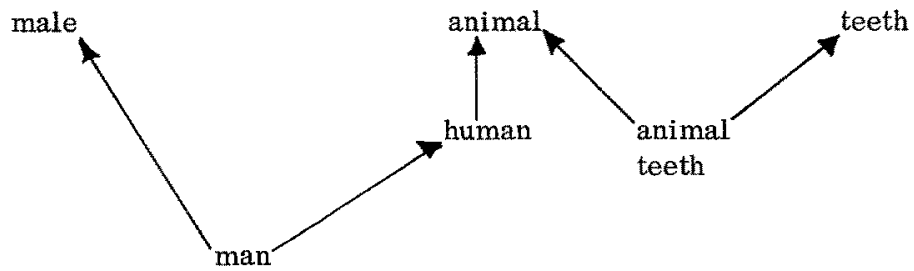

FIG. 2. The Representation of Intersection Concept Trees.

A single property may have more than one concept tree associated with it. For example, the properties "pet", "bird", and "fish" all imply the property "animal". But "animal", "bird", and "fish" are part of one concept tree while "animal" and "pet" are part of another concept tree. This can be seen from the fact that two properties which are subordinates within the same concept tree are mutually exclusive, i.e., a bird can not be a fish. But a bird can be a pet, thus their underlying concepts must be distinct. Logical Decision Making in the Formal Model.

It will be apparent that many of the definitions of relations are really theorems which can be proved from a few simple axioms which define the model. But it is not the purpose of this paper to present mathematical proofs, so all relations will simply be defined without attempting to distinguish which ones can be derived.

Axioms.

1. There exists a set $P$ of properties, such that $P=\left(p_{1}, \ldots, p_{n}\right)$, where $p_{i}$ is a property.

2. A concept tree $T_{x}$ is a lattice like the one shown in Figure 1 , where if $p_{j}$ is below $p_{i}\left(p_{j} \in T_{x}\left(p_{i}\right)\right)$, then $p_{j}$ implies $p_{i}$. For example, in Figure 1, since "human teeth" is below "animal teeth", "human teeth" implies "animal teeth".

3. A word is defined as a nonempty set of properties: for all $i, w_{i} \subset P$ and $w_{i} \neq 0$. A word must be defined as a set of properties to deal with the obvious but important fact that many words are ambiguous. For example, a club is both a weapon and a social organization. If a one-to-one mapping existed between words and properties then club would imply "concrete" and not imply "concrete", which is a contradiction.

\section{Definition of Relations.}

R1. Definition of "All" relations:

"All $w_{i}$ are $w_{j}$ "if there exists a $p_{i} \in w_{i}$ and a $p_{j} \in w_{j}$ such that $p_{j} \in T_{x}\left(p_{k}\right) \rightarrow p_{i} \in T_{y}\left(p_{k}\right)$. 
This says that if $p_{i}$ implies every property that $p_{j}$ implies, then the set which contains $p_{i}$ implies the set which contains $p_{j}$. For example, if a mammal contains the property "mammalian" which implies the properties "furry" and "animal" and dog contains the property "dog-like" which implies the properties "furry" and "animal" and "barks", then a dog is a mammal. A special case of this definition is when $p_{i}$ implies $p_{j}$. It follows from the transitive nature of implication that $p_{i}$ then implies every property which $p_{j}$ does. For example, "A canary is a bird" is true because the property "cannary-like" implies the property "bird-like".

R2. Definition of "No" relation:

"No $w_{i}$ is a $w_{m}$ " if there exists $p_{i} \in w_{i}$ and $p_{m} \in w_{m}$ and $p_{k}$ such that $p_{i} \in T_{x}\left(p_{k}\right)$ and $\mathrm{p}_{\mathrm{m}} \in \mathrm{T}_{\mathrm{x}}\left(\mathrm{p}_{\mathrm{k}}\right)$ and $\mathrm{p}_{\mathrm{i}} \notin \mathrm{T}_{\mathrm{y}}\left(\mathrm{p}_{\mathrm{m}}\right)$ and $\mathrm{p}_{\mathrm{m}} \notin \mathrm{T}_{\mathrm{z}}\left(\mathrm{p}_{\mathrm{i}}\right)$. This says that if $\mathrm{w}_{\mathrm{i}}$ and $\mathrm{w}_{\mathrm{m}}$ both have properties which are subordinates on the same concept tree, they are mutually exclusive. For example, a canary can not be a robin, since a bird can not be both a robin and a canary. A bird can not be a fish, since an animal can not be both a fish and a bird.

R3. "Definition of "Some" relation:

"Some $w_{j}$ are $w_{i}$ " if there exists a $p_{i} \in w_{i}$ and $p_{j} \in w_{j}$ such that $p_{j} \in T_{x}\left(p_{k}\right) \rightarrow p_{i} \in T_{y}\left(p_{k}\right)$. This is the inverse of the "all" relation (R1). If a canary is a bird, then some bird is a canary. If all pets are animals, then some animal is a pet.

R4. Extension of "Some" relation:

"Some $w_{i}$ is a $w_{n}$ " if there exists $p_{i} \in w_{i}$ and $p_{n} \in w_{n}$ and $p_{k}$ such that $p_{i} \in T_{x}\left(p_{k}\right)$ and $p_{n} \in T_{y}\left(p_{k}\right)$. This can best be explained by example. If some animal is a pet by R3 and a bird is an animal by R1, then since bird implies animal and some animal is a pet, some bird might be a pet. The crucial distinction here is that the properties of bird and pet are related to the property of animal under different concept trees $\left(T_{x}\right.$ and $\left.T_{y}\right)$. If they were part of the same concept tree, then R2 would apply. Of course, R3 and R4 define different relations. $R 3$ defines the existence of something, while $R 4$ only defines the possibility that something might exist. But this distinction is absent in normal English so it has been glossed over here.

R5. Definition of "Not all" relation:

"Not all $w_{j}$ are $w_{n}$ " if there exists $p_{i} \epsilon w_{i}$ and $p_{j} \epsilon w_{j}$ such that "All $w_{i}$ are $w_{j}$ " and $p_{n} \in w_{n}$ such that "No $w_{i}$ are $w_{n}$ ". This conjunction of $R 1$ and $R 2$ says that if a canary is a kind of bird and a canary can not be a robin, then not all birds are robins. Or, if there exists a bird which is not a pet, then not all animals are pets.

The Acquisition of Semantic Properties.

The representation just described is generated by adding new properties to concept trees. Clearly, by the definitions of the relations $\mathrm{R} 1-\mathrm{R} 5$, all logical relationships between 
the property added and every other property in the network are automatically specified. Previously, the problem arose of how to infer abstract logical implication from the representation of perceptual features. But here logical implication is defined a priori, so the problem never arises. It only remains to be shown that this logical system can have perceptual referents. A simple network labelled with its perceptual referents is shown in Figure 3. Note that the property "elm" implies both the perceptual features which make an

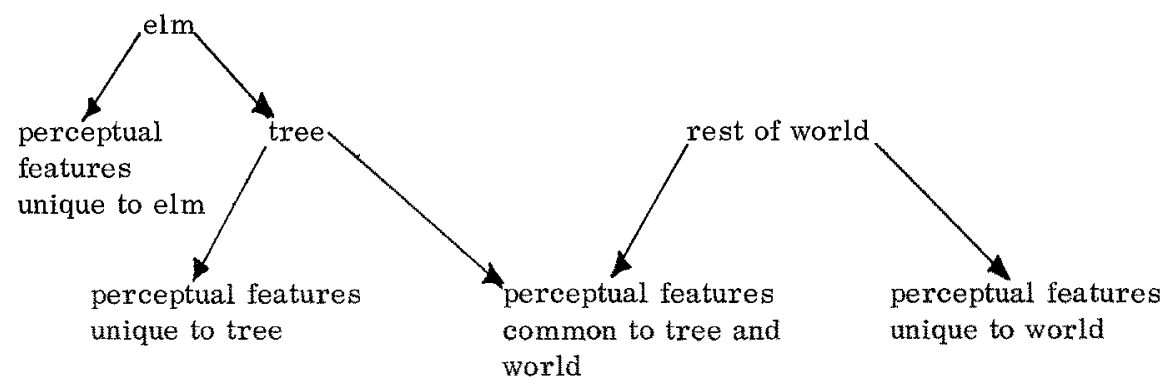

FIG. 3. Representation of How Logical Network Refers to Perceptual Data.

elm unique and the property "tree", which implies the features which make a tree unique. Hence, the property "elm" implies all the perceptual features of an "elm tree". This representation could have been generated in the following manner. A person saw a tree which he wanted to distinguish from all other trees. So a property (labelled "elm" in Figure 3) was attached to "tree". Thus an elm is a tree. Then an implication was drawn from "elm" to the perceptual features which an "elm" possesses in addition to those unique to all trees. Thus, logical implication can be related to its normal use in discriminating perceptual classes.

\section{Disambiguation Sentences.}

The theory just described can be used to clear up problems in other applications where a componential analysis has run into difficulty. For example, Katz and Fodor (1963) represented the properties defining the word bachelor by the tree structure shown in Figure 4. In the tree in Figure 1, the nonterminal properties at the branching points of the tree, which various meanings of bachelor have in common, are called markers, while the descriptions in brackets at the bottom of the tree, which are unique to each meaning of bachelor, are called distinguishers. The basic problem with ordering meaning in a hiexarchichal fashion is that it misses certain generalizations. The meanings of unmated seal and unmarried man certainly have something in common, but they are placed at the extreme ends of the tree. The academic degree holder and young knight share in common the fact that they have had titles bestowed upon them, but that fact is lacking from the tree. But if the markers were 


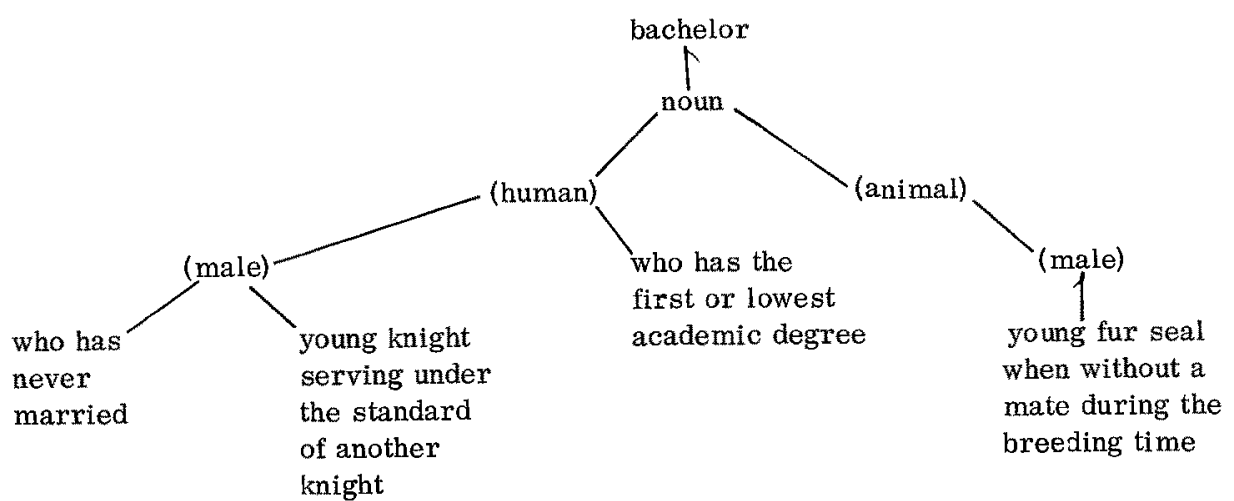

FIG. 4. : The Representation of the Meaning of Bachelor by Katz and Fodor.

rewritten and reordered to capture these generalizations, then the ones presently represented would be lost. Though language meaning is tantalizingly hierarchical, it is just crossreferential enough to vitiate an attempt to capture meaning in strict hierarchies. The representation of bachelor in this relationally defined theory is shown in Figure 5. As can be seen

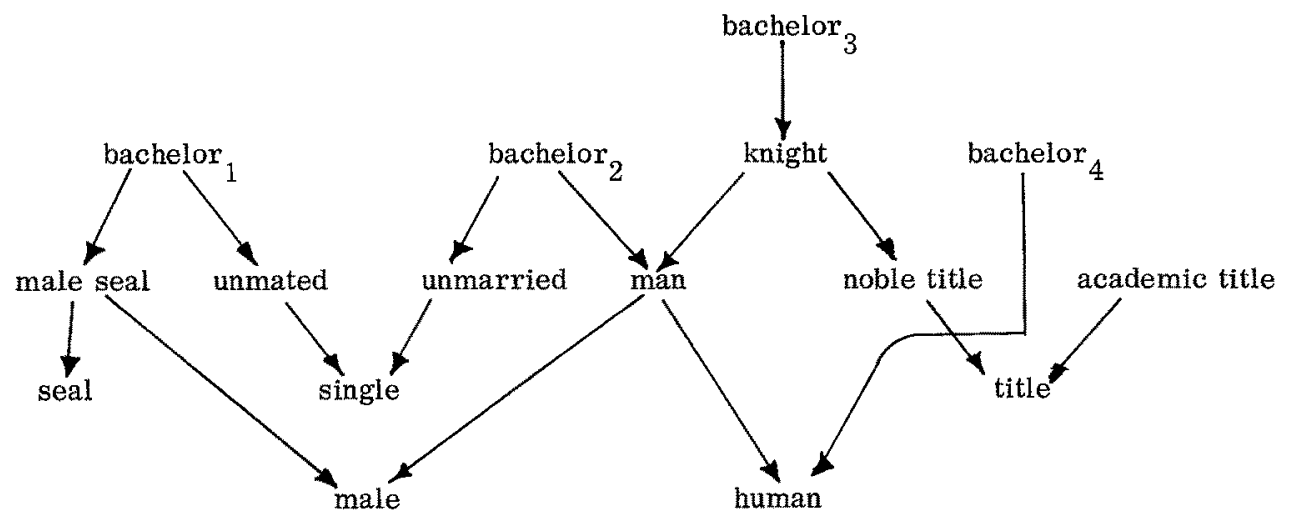

FIG. 5.: The Representation of the Meaning of Bachelor in the Relational Theory.

from the figure, the relational theory is more cross-referential in nature than the hierarchical theory Katz and Fodor propose. Further, there is a natural way of dealing with the disambiguation of a word in the context of a sentence. Disambiguation can take place by taking the intersections of the concepts associated with the properties defining the words in the sentence. For example, for "the barking bachelor", an intersection is sought with another concept tree which produces a logical relationship between a property of the concept "bark" and a property of bachelor. There is certainly an intersection between "bark" and 
"seal" since a seal bark is a bark and a barking seal is a seal. "Bachelor " is related to "seal" by $\mathrm{Rl}$ (a bachelor is a seal). Thus, by $\mathrm{R} 4$, "bachelor" is related to "barking seal" (a "bachelor" is a "seal" and some "seal" is a "barking seal"). Since the concept "bark" does not intersect any other concept tree of bachelor, the meaning is completely disambiguated. Thus, within the axioms of the theory, is a complete model of disambiguation.

\section{Syntactic Form and Semantic Meaning}

The theory can also be applied to the study of the relationship between syntax and symantics. Sintactic rules can be defined as relations between properties, similar to R1-R5. A sentence would then be a collection of several sets of pxoperties defining words with some of these relations defined between their properties. The following is a very simple example of this.

The distinction between analytic and synthetic is well-known. An analytic statement is one which is true by definition. For example, "All men are male" is analytic since part of the definition of being a man is being male. In contrast, while the statement "All men are over one inch tall" is nonetheless true, it is a synthetic statement since being over one inch tall is not part of the definition of man, and the sentence can only be verified in reference to the world. This semantic form of the analytic-synthetic distinction rests not on the syntactic relationship of the words in the sentence but simply on their meanings. But there is a second, syntactic form of the analytic-synthetic distinction in English. This rests solely on the syntactic rules which govern the construction of the sentence. To see this, note that sentence (i) is somehow odd, or peculiar, or ungrammatical.

(i) * A roommate is a writer.

But sentences (ii) and (iii) are perfectly acceptable.

(ii) My roommate is a writer.

(iii) A roommate is a person.

There seems to be something about the form of (i) that compels its interpretation as a universal assertion, independently of the meaning of the words concatenated. To see why this is so, some rules of sentence formation must be specified. The rules are given in extremely simplified form, with most of the details left out since they are not relevent to this example.

S1. The consistency rule.

Syntactic rules will be used to define new relations between properties. But if new implications are to be drawn between properties, they can not contradict the ones that already exist. It follows not only from this but any formal model, that a structure which leads to a logical contradiction is ill formed. This rule may be stated: for all $p_{i}$ and $p_{j}$ contained in $P$, if $p_{j}$ implies $p_{i}$ or $p_{j}$ and $p_{i}$ imply $p_{k}$, then $p_{i}$ can not imply $p_{j}$. 
S2. "A(n)" operator.

A syntactic rule has two parts to it. One part expresses a relationship which should exist or which should be generated between two properties, the same way the earlier relation rules specified relations between properties. The other part tells what words these properties are associated with by specifying the form of the word and where in the sentence it may be found. For example, the "A(n)" operator defines an implication: $p_{i} \rightarrow p_{a}$ where $p_{a}$ is a property of the set defining $\underline{a(n)}$ and $p_{i}$ is a property of a word $w_{i}$ following $\underline{a(n)}$ in the sentence.

S3. The "Is" operator.

The presence of $\underline{\text { is }}$ in a sentence defines an implication: $p_{i} \rightarrow p_{j}$ where $p_{i} \in w_{i}$ which precedes $\underline{\text { is }}$ and $p_{i} \rightarrow p_{a}$, and $p_{j} \in w_{j}$ where $w_{j}$ defines a word which follows is, In traditional grammatical terms, $w_{i}$ will be the noun and $\underline{a(n)}$ will be the determiner and the structure that contains $p_{i} \rightarrow p_{a}$ will be the noun phrase. Thus, $S 3$ says that in a sentence of this type, the noun phrase implies its predicate. We have seen from our three examples that this is true.

By application of $\mathrm{S} 2$ to the phrase a roommate, $\mathrm{p}_{\text {roommate }} \rightarrow \mathrm{p}_{\mathrm{a}}$ is defined. By application of $\mathrm{S} 3$ to the phrase a roommate is a writer, $p_{\text {roommate }} \rightarrow p_{\text {writer }}$ is defined. From the definition of $R 1$, this gives "All roommates are writers". But if the listener has stored a property implying $\mathrm{p}_{\text {roommate }}$ and sharing a concept with $\mathrm{p}_{\text {writer }}$ so that by $\mathrm{R} 5$ the listener knows "Not all roommates are writers", then a contradiction exists and by $\mathrm{S} 1$ the sentence must be ill formed.

Semantic Theory and Psychological Reality

The basic experimental paradigm to which this theory will be fitted was developed simultaneously by Landauer and Freedman (1968) and Collins and Quillian (1969). A subject is first presented with a semantic category, such as bird, and then a second word which may ox may not be an instance of that categroy. The subject presses a button labelled true if the instance word is a word like canary, and a button labelled false if the word is a word like rock. The dependent measure is how long it takes the subject to respond from the onset of the instance word. A second way this task is done is to present the entire assertion as a sentence: "A canary is a bird" and measure reaction time from the onset of the assertion. There have been no interesting differences reported from varying the form of the task (Kintach, et al, 1970) but the second version of the task is slightly more general. For example, one can also present a sentence which reads "A canary has wings". However, though sentences of this type are often reported to follow the same general pattern of experimental results as those where the assertions are restricted to the set-inclusion type (All A 
are $B$; Some $A$ are $B$ ), there are many contradictions in the data reported for them. Since it is not known why the data for these sentences is unclear, they will not be discussed.

The initial results found demonstrated that there were reliable differences in the time it took to respond true and false to different assertions. In general, it was found that a sentence of the type (1) "A canary is a bird" is responded to faster than a sentence of the type (2) "A canary is an organism". At first this was thought to reflect something about the logical form in which the meanings of the words were stored. For example, it was though that (1) was responded to faster than (2) because in order to respond to (2) the subject had to make more inferences. For (1), the subject would have stored directly the implication: canary $\rightarrow$ bird. But for (2) the subject would have to search through a network of implications: canary $\rightarrow$ bird $\rightarrow \ldots \rightarrow$ organism. However, since then, substantial evidence has accumulated that reaction time is not correlated with any measure of a chain of logical implications, but with the intuitive semantic distance between two words (Rips, Shoben and Smith, 1972). For example, a person is much more likely to think of a dog as an animal than a dog as a mammal. Dog and animal are intuitively close. If a subject is asked to respond to (1) "A dog is an animal" and (2) "A dog is a mammal", (1) is faster than (2) in spite of the fact that the chain of logical implications goes dog-mammal-animal. Semantic distance scales can be constructed by simply asking subjects to rank what words are intuitively close together or (Heider, 1972) by asking them to rate good and bad instances of a particular category. Reaction time correlates closely with intuitive distance on these scales and the seales correlate closely with each other. It seems that whether the question is how close the instance is to the category, or how good an example it is of that category, the same question is really being asked. When logical distance is held constant (A canary is a bird vs. A chicken is a bird) the better instance of the category (canary) is responded to faster. Semantic distance is correlated with conjoint frequency and associative propability. Conjoint frequency is a word count measure of how frequently two words appear together in the language. Associative propability is the frequency with which one word elicits the other as a response. It is reasonable to find that these measures are correlated with intuitive distance, since if words are found together one would expect them to be thought of together, and if words are thought of together, then one would expectethem to be produced together. However, the precise mechanism of the cause-effect relationship between acquisition, intuition, and production is still not known. But all such measures correlate well with reaction-time measures, to the detriment of logical distance models. The basic data may be summarized as follows. For statements which are true, the more intuitively similar the eategory and instance are, the faster the response. For statements which are false, the 
more intuitively similar the category and instance are, the slower the response. For example, "A canary is a dog" is responded to slower than "A canary is a flower".

In psychology, there is a peculiar distinction between what might be called empirical validity versus explanatory adequacy. For example, a way of handling the true/false data just presented would be to postulate that two separate processes are involved in verifying whether an instance is a member of a particular category. One of these processes is effective when there is no overlap between the properties of the words in the assertion, and both are effective where there is overlap. It is possible to contrive a multiple, serial, setsearch model that accounts for most of the experimental results. Such a model would be said to have empirical validity, since it predicts experimental results. Such models have been proposed, but when they have been proposed, not the slightest attempt has been made to show, in a noncircular manner, how the logical relationships between the words are inferred from the properties found. For example, paths between nodes may be defined which are labelled "set-relation". Then, to verify that a canary is a bird, one would look for a path labelled "set-relation" running from a node labelled "bird" to a node labelled "canary". Coding an English sentence as a labelled diagram cextainly does not constitute an explanation of it. Since such a theory really does not constitute an explanation of how a logical decision is made, it lacks explanatory adequacy. The fact that the theory presented here has attempted to deal with the question of explanatory adequacy distinguishes it from the other semantic reaction time models in psychology. It will now be shown to be empirically valid as well.

The approach that will be taken here is that what has been called intuitive distance or intuitive similarity is the causal factor underlying the differences in reaction-time in responding to different assertions. So it will first be shown how the theory can be made to account for semantic distance, and how that manipulation also accounts for reaction-time results.

The person who has most thoroughly discussed semantic distance is Eleanor Heider (1972). She points out that a word like bird often implies not only the properties of all birds, but the properties which most birds share. In other words, bird usually refers to a small, feathered animal, which lays eggs and sings. These can all be represented as properties of instances of birds. For example, the sentence "the birds were flying through the air" is much more likely than the sentence "the birds were running across the plain". But for an ostrich, it is the second sentence which is true. It appears that instances intuitively close to the category bird share most of the properties it subtly connotes. For example, sparrow, robin, wren, and canary can all be described adequately as small feathered animals which fly and sing. A few additional properties, such as "yellow" for canary, may define each 
bird uniquely, but these properties are additive, not alternative, to the ones mentioned. But a bad instance, such as ostrich, requires many additional properties in its definition, since it is large, does not fly, and does not sing. Similarly, a chicken cackles, does not fly, and is raised for eggs and meat. Thus, the measure of intuitive semantic distance will be how many additional properties it takes to define the instance over the property which defines the category. For example, if a canary is defined as a "yellow" "bird" and an ostrich requires three additional properties (large, nonflying, nonsinging, bird) to canary's one, then canary is intuitively closer to bird.

Though a word was defined as a set of properties, so far $w_{i}$ has been treated as a one-element set. In order to explain semantic distance, a word will now be treated as a multi-element set containing all the properties it logically implies. For example, instead of mammal being defined by the set containing the property "mammal", mammal will be defined by the set consisting of "furry", "animal", etc., which "mammal" logically implies. These two descriptions can be related by defining the sum of the branches of a property $B\left(p_{i}\right)$ as the set of properties such that $p_{j} \in B\left(p_{i}\right)$ if $p_{i} \rightarrow p_{j}$. The "furry" and "animal" are the branches of "mammal". The semantic distance between two words defined by $\mathrm{B}\left(\mathrm{w}_{\mathrm{i}}\right)$ and $B\left(w_{j}\right)$ can now be defined by the following formula:

$$
\text { Intuitive Distance }\left(\mathrm{I}_{\mathrm{w}_{i}, w_{j}}\right)=\frac{a}{b+c}
$$

where $a$ is the number of properties in $v_{a}$ and $b$ is the number of properties in $v_{b}$ and $c$ is the number of properties in $\mathrm{v}_{\mathrm{c}}$.

$$
\begin{aligned}
& p_{i} \in w_{i}, \quad\left(p_{j} \in w_{j}\right) \\
& p_{a} \in v_{a} \text { if } \quad p_{i} T_{x}\left(p_{a}\right) \wedge\left(p_{j} \in T_{y}\left(p_{a}\right) \vee p_{a} \in w_{j}\right) \\
& p_{b} \in v_{b} \text { if }\left(p_{b} \in B\left(p_{i}\right) \wedge p_{b} \notin B\left(p_{j}\right) \vee\left(p_{b} \in B\left(p_{j}\right) \wedge p_{b} \notin B\left(p_{i}\right)\right)\right. \\
& p_{c} \in v_{c} \text { if } p_{i} \in T_{x}\left(p_{c}\right) \wedge p_{j} \in T_{x}\left(p_{c}\right)
\end{aligned}
$$

For a word pair like cardinal/blue jay, $\mathrm{v}_{\mathrm{a}}$ consists of all the properties which have a tree containing a property of $w_{i}$ and another tree containing a property of $w_{j}$ associated with them. These are properties like "feathered", "beaked", "taloned", etc. v consists of properties not in common: such as the fact that a cardinal is a sport's emblem. $\nabla_{c}$ consists of properties which do discriminate the sets, such as the property "colored", since both "red" (for cardinal) and "blue" (for bluejay) are members of the same concept tree "colored" and hence, by R2, mutually exclusive alternatives. For a word pair like cardinal/bird the 
property "red" would be in $\mathrm{v}_{\mathrm{b}}$, since bird does not imply any color, and $\mathrm{v}_{\mathrm{c}}$ would be empty. It follows from the definition of "All" that $w_{c}$ must be empty whenever "All" is true. Also, if a property in cardinal implies a property in bird, such as "bird-like", then "bird-like" goes into $\mathrm{v}_{\mathrm{a}}$. The formula for intuitive distance: the ratio of the number of properties the words have in common $\left(v_{a}\right)$ to the number of properties not held in common $\left(v_{b}\right)$ plus the properties logically distinguishing the words $\left(\mathrm{v}_{\mathrm{c}}\right)$ follows closely the previous discussion of what the underlying basis of intuitive distance could be.

The relevent parameter for predicting reaction time is the percentage of properties in the union of the sets $\mathrm{B}\left(\mathrm{w}_{i}\right)$ and $\mathrm{B}\left(\mathrm{w}_{\mathrm{j}}\right)$ on which a decision about the relationship between the two words can be based. This stems from the assumption that the union of the word-sets is searched for a property on which to make a decision about their relationship. Where the percentage of such properties is larger, the probability of finding one is greater and the average time to make a decision should be shorter. In the case of a true statement like "A cardinal is a bird", $v_{c}$ will be empty. The larger $v_{a}$ is, the faster a decision will be reached; but by definition, the larger $v_{a}$ is the closer the two words will be intuitively. Thus, for true statements, the closer the words are intuitively, the faster the reaction-time. For false statements, like "A cardinal is a bluejay", the reaction time will depend on the percentage of properties in $v_{c}$. But where words are intuitively close, and $v_{a}$ is large, $v_{c}$ will be proportionally small. Thus, the closer the intuitive distance for false statements, the slower the reaction time.

It would be nice to have some more evidence for the existence of $v_{a}, v_{b}$, and $v_{c}$. Such evidence exists. Meyer (1970) had subjects respond true or false to such statements as (1) "All stones are rubies", (2) "All mothers are writers", (3) "All typhoons are wheats" and (4) "Some stones are rubies", (5) "Some mothers are writers" and (6) "Some typhoons are wheat". There are two possible ways a decision to respond false could be reached in (1), (2) and (3). One way is that, for example, a $p_{b} \in B$ (rubies) could be found so that the requirement of the "All" relation that all $\mathrm{p}_{\mathrm{k}}$ implied by $\mathrm{p}_{\text {rubies }}$ are implied by $\mathrm{p}_{\text {stone }}$ could not be satisfied. The other way is that, for example, a $p_{c}$ for an instance of mother and writer could be found so that the requirements of the "Not all" relation sould be satisfied. It does not matter which property is found since predictions about the speed of reaction time are based on the ratio $b+c / a$ and are thus independent of whether the falsifying property is $a p_{b}$ or $p_{c}$. Clearly, stone/ruby are intuitively closer than mother/writer, which are intuitively closer than typhoon/wheat: so (1) should be slower than (2) which should be slower than (3). The actual average reaction times were 1339 milliseconds (msec.) for (1), 1263 msec. for (2) and $1154 \mathrm{msec}$. for (3), which are highly significant differences $(p<.001)$. The results are exactly as predicted. 
For "Some typhoons are wheats" the false decision still rests on a property in $v_{b}$ or $\mathrm{v}_{\mathrm{c}}$, so the reaction time for it should not differ from the "All" statement. The measured time of 1115 msec. does not differ significantly ( $p .10)$ from 1154 msc. But for (4) "Some stones are rubies" and (5) "Some mothers are writers" a decision will be arrived at by findi ng a $p_{a}$. Clearly, if $b+c / a$ is greater than $b^{\prime}+c^{\prime} / a^{\prime}$, then $a^{\prime} / b^{\prime}+c^{\prime}$ will be greater than $b+c / a$. Thus the theory predicts that (4) will now be faster than (5). It is also intuitively sound to expect that (5) will be faster than (2), since mother/human intuitively has more properties in common than not in common. The reaction times are, for (4), $1017 \mathrm{msec}$. and for (5), 1108 msec., which is significantly faster (p .01) than the time for (2), 1263 msec. Thus, once again all predictions are confirmed (see Fig. 6).

$$
\begin{array}{r}
1350 \\
1300 \\
1250 \\
1200 \\
1150 \\
1100 \\
1050 \\
1000 \\
950
\end{array}
$$$$
\text { ALL }
$$

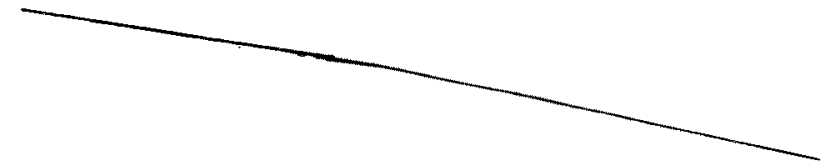
950

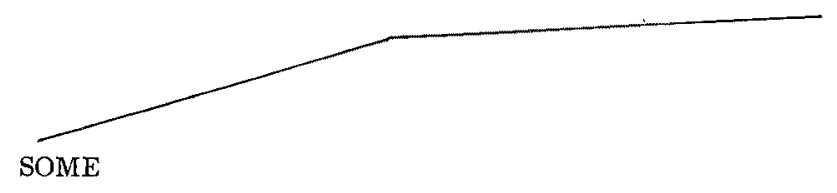

$$
\text { stone/ruby mother/writer typhoon/wheat }
$$

FIG. 6. Reaction Time Results from Meyer (1970).

There is also evidence that the "All" and "Some" relations are correctly defined. An "All" decision can depend on finding several properties in $\mathrm{p}_{\mathrm{a}}$, but for a "Some" decision only a single property need be found in $p_{a}$. Thus, "All chairs are furniture" should be slower than "Some chairs are furniture". Their times are $1182 \mathrm{msec}$. and $990 \mathrm{msec}$. $(\mathrm{p}<.001)$, respectively.

\section{Conclusion}

Traditional componential analysis has been applied to a wide range of semantic problems. In this paper logical implication has been proposed as an alternative to setinclusion as the primitive out of which meaning is built. It has been shown to apply to an equally wide range of problems as theories utilizing set-inclusion, but with the possibility of even greater success. The theory is potentially applicable to the study of logic and syntax, of language acquisition, and the investigation of the organization of memory through reaction time. 


\section{Acknowledgment}

Part of the work on this paper was supported by a grant from the Undergraduate Research Council of the State University of New York at Buffalo. I would like to thank John Anderson and Gordon Bower for their time and effort in reading and commenting on the many versions of this paper.

\section{References}

Bollinger, D. (1965), "The Atomization of Meaning", Language, 41, 555-573.

Collins, A.M. and M.R. Quillian (1969), "Retrieval Time from Semantic Memory", Journal of Verbal Learning and Verbal Behavior, , 24-248.

Heider, E.R. (in press), "On the Internal Structure of Perceptual and Semantic Categories", in T.M. Moore (ed.) Cognitive Development and Acquisition of Language, New York, Academic Press.

Katz, J.J. and J. Fodor (1963), "The Structure of a Semantic Theory", Language, 39 , 170-210.

Kintsch, W., E. Crothers and L. Berman (1970), "The Effects of Some Semantic and Syntactic Properties of Simple Sentences upon the Latency of Judgements of Semantic Acceptability", in Studies in Mathematical Learning Theory and Psycholinguistics, The University of Colorado.

Landauer, T.K. and J.L. Freedman (1968), "Information Retrieval from Long-Term Memory: Category Size and Recognition Time", Journal of Verbal Learning and Verbal Behavior, 7, 291-295.

Lyons, J. (1969), Introduction to Theoretical Linguistics, London, Cambridge University Press.

Meyer, D.E. (1970), "On the Representation and Retrieval of Stored Semantic Information", Cognitive Psychology, $1,242-300$.

Rips, L.J., E.J. Shoben and E.E. Smith (1972), "Semantic Distance and the Verification of Semantic Relations", unpublished manuscript.

Schaeffer, B. and R. Wallace (1970), "The Comparison of Word Meanings", Journal of Experimental Psychology, 6, 144-152.

Schank, R.C. (1971), "Intention, Memory, and Computer Understanding", Stanford Artificial Project Memo AIM-140.

Wilkins, A.T. (1971), "Conjoint Frequency, Categroy Size, and Categorization Time", Journal of Verbal Learning and Verbal Behavior, 10, 382-385.

Winograd, T. (1972), "Understanding Natural Language", Cognitive Psychology, $\underline{3}, 1-191$. 